\title{
RUTA CULTURAL - MÉRIDA, EDILICIA MODERNA: LA VIVIENDA UNIFAMILIAR
}

\section{Giselle A. Arnal Sandia ${ }^{9}$}

${ }^{1}$ Universidad de Los Andes. Facultad de Ingeniería, Escuela Básica, Dpto. de Cs. Aplicada y Humanística. Mérida - Venezuela

Autor para correspondencia: herearnal@gmail.com

Recibido: 2020/03/11

Aprobado: 2021/05/25

DOl: https://doi.org/10.26621/ra.v1i24.670

\section{RESUMEN}

Una comunidad empoderada de su patrimonio es aquella que se ha hecho responsable del mismo y aboga por su conservación y preservación. Es por ello que en la actualidad, diversas instituciones importantes invitan a la creación de estrategias que permitan a la comunidad empoderarse de su patrimonio. Mérida - Venezuela es una ciudad privilegiada ya que sus características geográficas, históricas y sociales la hacen una candidata perfecta para desarrollar una sinergia de turismo y cultura. La creación de una ruta cultural de los bienes inmuebles, de corte artístico - tipología residencial denominada Mérida Edilicia Moderna - la vivienda unifamiliar, basada en la definición de rutas culturales, usando como recurso a las viviendas de corte moderno, permitirá a los usuarios hacer un recorrido diferente por el casco central de la ciudad, conociendo así al hombre merideño de mediados del siglo XX. Este resultado evidencia una alternativa donde la ciudad de Mérida deja de ser la ciudad tradicional del damero americano, para convertirse en una ciudad que entra en la modernidad mundial.

Palabras clave: patrimonio arquitectónico, rutas culturales, turismo cultural.

\section{ABSTRACT}

A community that It is empowered of its heritage It has assumed the responsibility of its conservation and preservation. This is why, in our days, different important institutions, invites the community to create strategies that It allows them to be empowered regarding its heritage. Merida, is a privileged city, for its geographic, historical and social qualities makes her a perfect candidate to create a cultural and tourism synergy. The creation of a cultural route named Merida Edilicia Moderna - la vivienda unifamiliar, based on the definition of cultural route, using the modern houses from the downtown as resources, it will allow users to get to know the city's downtown in a different way, meet Merida's men from mid XX c. and how Merida stops being the traditional American checkerboard city to become a city on the verge of global modernity.

Keywords: architectural heritage, cultural routes, cultural tourism 


\section{INTRODUCCIÓN}

La ciudad de Mérida - Venezuela es rara vez asociada con hechos arquitectónicos de gran relevancia. Por lo general, el conocimiento popular sobre arquitectura se limita a reconocer las "casas coloniales", la cuadra Arzobispal (incluyendo la Basílica Menor) y el Edificio Rectoral de la Universidad de Los Andes. Cuando se intenta mencionar obras relevantes de la ciudad, pareciera que el quehacer arquitectónico se estancó en una labor de construcción por la mera razón de cubrir necesidades. Y es que, tanto Mérida como la propia Venezuela, no poseían hechos de mayor preeminencia arquitectónica hasta bien entrado el s. XX. A finales del s. XIX, Mérida fue descrita por un viajero de la siguiente manera: "El aspecto material de la población es bueno y presenta un conjunto regular, sin que, por otra parte, se descubra nada notable, ni tampoco particular esmero o variedad en las construcciones. La mayor parte de las casas son bajas, con ventanas grandes, estilo que predomina en casi todo Venezuela" (Laverde Amaya citado en Moreno Rodríguez \& Bhaszar, 2020).

Sin embargo, Cherini Ramirez (2007) afirma que la ciudad de Mérida posee un pasado que merece ser perpetuado, por su diversidad y riqueza La ciudad de Mérida, que cuenta con más de 460 años, es una ciudad "abundante en historia y riquísima en tradición, acumuladas durante cuatro centurias [...] Por calles de la Ciudad -entonces polvorientas y empedradas- pasaron conquistadores, colonizadores, libertadores y caudillos; algunas casas en ella son lugar de natal o morada de escritores, patricios, educadores, religiosos y prelados; la Administración Pública, hispánica y republicana, ha dejado aquí sello de actuaciones importantes, diversos sucesos trascendentales ocurrieron en el recinto del Ayuntamiento, del Seminario San Buenaventura, La Universidad Emeritense, y en otros edificios de la ciudad prócera" (Febres Cordero citado en Cherini Ramírez, 2007).

De igual forma, al observar con mayor profundidad la retícula urbana de la ciudad, es posible recordar una época e identificar vestigios de ella, en donde la ciudad de Mérida era centro de crecimiento y de experimentación de las vanguardias y del movimiento moderno. Este proceso se llevó a cabo en varias fases, siendo la primera irradiación en la ciudad del movimiento moderno durante la década de 1950 (Febres-Cordero, 2003). Mérida, a pesar de encontrarse en una ubicación geográfica menos favorable que otras ciudades venezolanas, tuvo un proceso de modernización a partir del segundo cuarto del siglo XX, impulsado mayormente por la iglesia católica y la Universidad de Los Andes (Arnal, 2016).

Nacido en Europa, el Movimiento Moderno consigue un espacio en las escuelas de arquitectura de este continente a través de las publicaciones realizadas en diversos medios, así como el recibimiento de sus grandes maestros en estas latitudes, quienes huían de la realidad política de sus países natales. Los mandatarios de muchos países latinoamericanos, en especial los de Venezuela, encontraron en los principios fundamentales del Movimiento Moderno una bandera política y de desarrollo para sus naciones.

Antonio Guzmán Blanco, en fecha tan temprana como finales del siglo XIX, utiliza el Movimiento Moderno para transformar arquitectónicamente la ciudad de Caracas iniciando el tránsito del país hacia la modernidad, con base en los ideales positivistas orden y progreso, lo que deja "una huella indeleble de la nación, superando momentos de crisis social y natural" (Moreno Rodríguez \& Bhaszar, 2020).
Arquitectos como Mujica Millán, Bosetti, Lluch, Cova Rey, Villanueva, Vivas, Sanabria, entre otros, hallaron en la ansiosa por modernizarse ciudad de Mérida un lugar para exponer sus ideas y ejecutarlas; ello dejó una marca tanto en la población como en la ciudad, convirtiéndose en parte fundamental de su patrimonio. Según Mora (citado en Cherini Ramírez, 2007), la basta intervención que realizó el arquitecto Mujica Millán en el casco central de la ciudad, con la creación de una nueva tipología que nace de la reinterpretación del estilo colonial, "logró valorizar la ciudad desde el punto de vista urbano, proporcionándole una imagen significativa. Al mismo tiempo, estos nuevos edificios comienzan a conformar el patrimonio cultural de la ciudad del siglo XX".

Ahora bien, la preservación y conservación del patrimonio inmueble de la ciudad de Mérida ha enfrentado una serie de debilidades que ha puesto en riesgo su supervivencia y protección para el futuro. El principal problema parece ser el desconocimiento de la población de la existencia del mismo, así como la falta de puesta en valor y de gestión por parte de los entes competentes.

Por sus características geográficas, históricas y sociales, la ciudad de Mérida es candidata para la creación de una dinámica de rutas culturales como propuesta para la puesta en valor de las edificaciones patrimoniales existentes en ella (Arnal, 2016). La creación de una ruta cultural cuyo hilo conductor sea el Movimiento Moderno permitirá dar a conocer a la comunidad en general, así como a un público más especializado, la existencia de obras arquitectónicas de este movimiento en la ciudad.

El rol que jugaron la iglesia y la Universidad de Los Andes en la transformación de la ciudad de Mérida puede ser resumido con la afirmación de Chacón (2015) "Hablar del seminario es hablar de la universidad y hablar de la universidad es hablar de Mérida"; Comprender esto, así como la adecuación del movimiento moderno a la ciudad de Mérida, es una necesidad y un primer paso para poder valorar y difundir las diferentes obras realizadas en la ciudad por los exponentes de dicho movimiento. Esto permitirá, posteriormente, crear otras estrategias que permitan preservar estas edilicias como patrimonio emeritense, e incluir dentro del catálogo de patrimonio del Municipio Libertador aquellas obras de la arquitectura moderna que no gozan de declaratoria, muy particularmente las viviendas de la periferia del casco central.

Una serie de rutas culturales, para Mérida, fueron propuestas utilizando la metodología de López (2006), quedando algunas definidas y otras pre - diseñadas (Arnal, 2016). Esta metodología consta de seis pasos: justificación, objetivo general, objetivos secundarios, inventario de los recursos e información, estudio del mercado potencial, y creación de la ruta (en la cual se desarrolla el recorrido, los convenios, el guión, la imagen, etc.).

Tras la realización de un inventario, que permitió reconocer y clasificar los elementos patrimoniales, fue posible establecer una ruta denominada Mérida Edilicia Moderna: Ia vivienda unifamiliar, la cual agrupa los bienes patrimoniales clasificados en los hilos conductores Época Moderna Contemporánea - etapa I y II y Edificaciones Civiles Residenciales (Arnal Sandia, 2016).

\section{MÉTODOS}

\section{Turismo cultural, rutas culturales y patrimonio}

El éxito en la conservación y preservación del patrimonio está estrechamente ligado a que la comunidad se empodere del mismo. Es por ello que ICOMOS (Consejo Internacional de Monumentos y Sitios, por sus siglas en inglés), a través de la Declaración de Florencia (2014), recalca la necesi- 
dad de comprometer a la comunidad con su patrimonio e invita a desarrollar propuestas participativas que permitan apreciarlo, así como proteger la integridad y autenticidad del mismo y de sus tradiciones. ICOMOS (2014) afirma que solo será posible lograr una comunidad empoderada a través de actividades como las anteriormente mencionadas.

Como una opción para dar a conocer la cultura, nace el concepto de turismo cultural. Actividades como las rutas culturales "son uno de los recursos más frecuentes a los que recurren los organismos públicos y los operadores turísticos para promocionar un destino o vender un producto" (Rengifo, 2006), incluidos el patrimonio cultural y natural de dicho destino.

El concepto de itinerario cultural varía según diferentes organismos, en vista del enfoque que cada uno le otorga al concepto; aun cuando parten de la misma raíz y objetivo (preservar el patrimonio), los conceptos pueden diferir el uno del otro. Para el caso particular de este trabajo de investigación, las rutas culturales son entendidas "como propuestas de recorridos especiales de contenido patrimonial que sirven como ejes de difusión de la relación cultura - territorio - identidad" (Carrera, 2006).

Siendo las rutas culturales un instrumento "para acercar el patrimonio a la sociedad" (López, 2006), a la hora de diseñar una o varias de ellas, es necesario tomar en cuenta que este es un trabajo multidisciplinario, que las mismas deben tener un tema principal del que puedan surgir temas secundarios, y deben estar dirigidas tanto a expertos en el área de patrimonio como a la población común; pero, sobre todo, la ruta debe ser portadora de un significado cultural (Arnal Sandia, 2016).

A fin de evitar un aglutinamiento y un discurso disperso a lo largo del recorrido, y considerando que existe una diversidad ingente de recursos patrimoniales que pueden ser incorporados a una ruta cultural, López (2006) considera "conveniente identificar ejes temáticos que faciliten la comprensión del patrimonio", pudiendo clasificarse de la siguiente manera:

1. Territorial o geográfico.

2. Temas históricos.

3. Aspectos artísticos.

4. Criterios literarios.

5. Actividades económicas.

6. Etnografía: en este apartado las rutas culturales suelen basarse en el patrimonio cultural intangible: arte popular, tradiciones y costumbres, museos etnográficos y fiestas populares.

7. Patrimonio cultural: "En este apartado incluimos aquellas rutas que por su naturaleza o carácter son difíciles de encajar en los temas señalados, ya que pueden aglutinar en una misma ruta diversos recursos o ser el complemento de otras rutas" (López, 2006, p. 31).

Comprender las implicaciones que tiene el hecho de que "las rutas culturales se integran fácilmente, incluso de manera espontánea, dentro de lo que se suele llamar turismo cultural" (Torres, 2006), es fundamental para que una ruta cultural funcione a plenitud. En palabras de López (2006): "desde el punto de vista turístico (...) son un instrumento para facilitar a los visitantes el conocimiento de una ciudad (...) que habitualmente tienen un hilo conductor de carácter cultural".

Apegadas a la memoria, tradición y a la naturaleza, las rutas culturales pueden ser exitosas y cumplir con su objetivo de puesta en valor del patrimonio. De acuerdo con el mismo autor, si tienen un motivo central, presentan una oferta diversificada, cuentan con publicidad, ofrecen accesibilidad física y cognitiva, brindan beneficios económicos y cuentan con un equipo interdisciplinario.
Procedimientos de clasificación y registro de los bienes patrimoniales cada vez más estandarizados permiten diseñar, en la actualidad, y con mayor eficacia, rutas culturales. Estos procedimientos "permiten seleccionar y objetivar aquellos bienes culturales y ecológicos que son susceptibles a transformarse en productos turísticos, organizando y jerarquizando la oferta según su interés turístico" (Hernández, 2011); siempre recordando que las rutas culturales se basan en un apego a la memoria, tradición y naturaleza.

Según López (2006), para que un itinerario o ruta cultural sea exitoso y cumpla su objetivo de puesta en valor del patrimonio, debe cumplir con las siguientes características:

1. Tener un motivo central que permita alcanzar el objetivo principal de dar a conocer y difundir el patrimonio.

2. Presentar una oferta diversificada.

3. Contar con publicidad que le permita llegar al mercado.

4. Ofrecer accesibilidad física (monumentos abiertos y medios para discapacitados) y cognitiva.

5. Poseer beneficios económicos que conlleven a la conservación del patrimonio.

6. Ser interdisciplinario.

A fin de poder lograr lo anterior, y así proponer rutas o itinerarios culturales que permitan dar a conocer el patrimonio de la mejor manera posible, López (2006) plantea una metodología multidisciplinaria a seguir mostrado en la Tabla 1.

\section{RESULTADOS Y DISCUSIÓN}

\section{Ruta Cultural: Mérida Edilicia Moderna - la vivienda unifamiliar}

Esta investigación partió de un trabajo previo (Arnal Sandia, 2016) que permitió catalogar las edificaciones con valor patrimonial de la ciudad de Mérida con base al valor patrimonial que cada una posee, así como por características comunes que éstas puedan tener; ello se hizo con el fin de agrupar las edificaciones basándose en los hilos conductores, creando el corpus para posibles rutas culturales.

La investigación tuvo un enfoque proyectivo y constó de un diseño transeccional contemporáneo documental. En este caso en particular, de los seis pasos planteados por López (2006), se desarrollaron cuatro en su totalidad debido a la naturaleza de la metodología y del enfoque multidisciplinario e integrador que propone el autor (Tabla 2).

Fundamentado en incentivar la conciencia y la memoria colectiva de los habitantes, es importante que la población identifique y gestione, como comunidad empoderada, su patrimonio cultural. Esto conlleva a que aquellos lugares y edificaciones con valor patrimonial puedan recuperarse y preservarse para las generaciones futuras. Sin embargo, para ello es necesario que tanto los entes gubernamentales como las comunidades definan políticas y estrategias que apunten hacia la preservación de su patrimonio cultural.

Las características geográficas, históricas y sociales de la ciudad de Mérida permiten crear una dinámica entre la puesta en valor de las edificaciones patrimoniales (que presentan vestigios de otras épocas) y reflejos de su realidad, y el turismo presente en la ciudad. 
Tabla 1. Procedimiento de diseño de rutas culturales.

\begin{tabular}{lll} 
& PASOS & DESCRIPCIÓN \\
\hline 1 & $\begin{array}{l}\text { Justifica- } \\
\text { ción }\end{array}$
\end{tabular}

"En principio la mayoría de los proyectos debe tener como prioridad el fomento del desarrollo turístico y económico de una zona determinada sin olvidar la importancia y el

2 Objetivo valor del patrimonio que suele ser el recurso prioritario. General Cuando se plantea el objetivo debe adecuarse a la realidad, hay que tener muy claro para qué diseñamos nuestra ruta, qué pretendemos con ella" (p. 32).

Objetivos Aquellos objetivos que permitirán alcanzar el objetivo prin3 Secunda- cipal. rios

Inventa - "Antes de iniciar el trazado de la ruta es necesario conocer Inventa- los recursos con los que contamos y toda la información 4 Recursos que se tiene de ellos para realizar un inventario" (p. 33), e Informa- debería incluir un estudio del estado de conservación de e Informa- los mismos, viabilidad, relación entre los elementos y la
ción importancia de cada uno para el proyecto.

"Estudio del posible mercado, tanto desde el punto de vista Estudio del cualitativo como cuantitativo, a quién puede interesar, qué 5 Mercado tipo de turismo, la población local, las infraestructuras tuPotencial rísticas (hoteles y restaurantes, el porcentaje de ocupación que tienen), a qué tipo de turista puede interesar" (p. 33).

Accesibilidad

Concentración o no de los recursos

Acceso a la documentación e información

Creación de la ruta: establecer las etapas, explicación de las mismas, recorridos, etc.

6 Creación $\begin{aligned} & \text { Fechas aptas para la realización de la ruta } \\ & \text { de la Ruta }\end{aligned}$ completa o parte de la misma

\begin{tabular}{l}
\hline Financiamiento \\
\hline Imagen y logotipo \\
\hline Comercialización y divulgación \\
\hline Evaluación de procesos y resultados
\end{tabular}

Fuente: Arnal Sandia en base a López, 2016.

Existen un total de 86 bienes inmuebles con valor patrimonial en la superficie de la ciudad conocida como "casco central", desde la intersección de la avenida 1 Rodríguez Picón con la avenida Lora hasta el viaducto Miranda entre ambos bordes de la meseta, de los cuales 38 son de tipología residencial.

La tipología de vivienda unifamiliar es una de las más cercanas a la vida cotidiana del ciudadano merideño puesto que el diseño de las viviendas está netamente asociado con la vida íntima y personal de quien la habita, así como con las tendencias de la época. Es por ello que a través de las viviendas es posible realizar una lectura del comportamiento del hombre, sus oficios, necesidades y limitantes.
Tabla 2. Proceso de diseño de la ruta cultural.

\begin{tabular}{|c|c|c|c|}
\hline & PASOS & SUB - PASO & $\begin{array}{l}\text { NIVEL DE } \\
\text { EJECUCIÓN }\end{array}$ \\
\hline 1 & \multicolumn{2}{|l|}{ Justificación } & $\begin{array}{l}\text { Ejecutado: } \\
\text { Completo }\end{array}$ \\
\hline 2 & \multicolumn{2}{|l|}{$\begin{array}{l}\text { Objetivo } \\
\text { General }\end{array}$} & $\begin{array}{l}\text { Ejecutado: } \\
\text { Completo }\end{array}$ \\
\hline 3 & \multicolumn{2}{|l|}{$\begin{array}{l}\text { Objetivos } \\
\text { Secundarios }\end{array}$} & $\begin{array}{l}\text { Ejecutado: } \\
\text { Completo }\end{array}$ \\
\hline \multirow{3}{*}{4} & \multirow{3}{*}{$\begin{array}{l}\text { Inventario de } \\
\text { los Recursos e } \\
\text { Información }\end{array}$} & Inventario / recurso & $\begin{array}{l}\text { Ejecutado: } \\
\text { Completo }\end{array}$ \\
\hline & & Viabilidad de incorporación & $\begin{array}{l}\text { Ejecutado: } \\
\text { Completo }\end{array}$ \\
\hline & & Importancia & $\begin{array}{l}\text { Ejecutado: } \\
\text { Completo }\end{array}$ \\
\hline 5 & \multicolumn{2}{|l|}{$\begin{array}{l}\text { Estudio del } \\
\text { Mercado } \\
\text { Potencial }\end{array}$} & No ejecutado \\
\hline \multirow{10}{*}{6} & \multirow{10}{*}{$\begin{array}{l}\text { Creación } \\
\text { de la Ruta }\end{array}$} & Accesibilidad & $\begin{array}{l}\text { Ejecutado: } \\
\text { Incompleto }\end{array}$ \\
\hline & & $\begin{array}{l}\text { Concentración o no de los } \\
\text { recursos }\end{array}$ & $\begin{array}{l}\text { Ejecutado: } \\
\text { ncompleto }\end{array}$ \\
\hline & & $\begin{array}{l}\text { Acceso a la documentación e } \\
\text { información }\end{array}$ & $\begin{array}{l}\text { Ejecutado: } \\
\text { ompleto }\end{array}$ \\
\hline & & $\begin{array}{l}\text { Creación de la ruta: establecer las } \\
\text { etapas, explicación de las mis- } \\
\text { mas, recorridos, etc. }\end{array}$ & $\begin{array}{l}\text { Ejecutado: } \\
\text { Completo }\end{array}$ \\
\hline & & $\begin{array}{l}\text { Fechas aptas para la realización } \\
\text { de la ruta }\end{array}$ & $\begin{array}{l}\text { Ejecutado: } \\
\text { Completo }\end{array}$ \\
\hline & & $\begin{array}{l}\text { Temporalización: tiempo } \\
\text { necesario para realizar la ruta } \\
\text { completa o parte de la misma }\end{array}$ & $\begin{array}{l}\text { Ejecutado: } \\
\text { Completo }\end{array}$ \\
\hline & & Financiamiento & No ejecutado \\
\hline & & Imagen y logotipo & $\begin{array}{l}\text { Ejecutado: } \\
\text { Incompleto }\end{array}$ \\
\hline & & Comercialización y divulgación & No ejecutado \\
\hline & & $\begin{array}{l}\text { Evaluación de procesos y } \\
\text { resultados }\end{array}$ & No ejecutado \\
\hline
\end{tabular}

Fuente: Arnal Sandia, 2016.

Si bien la ciudad de Mérida rara vez es asociada con hechos arquitectónicos de gran relevancia, para muchos, el centro de la ciudad es un compendio de edificaciones coloniales a excepción de la cuadra Arzobispal y el Edificio del Rectorado de la Universidad de Los Andes. Sin embargo, quien mira con profundidad la ciudad y la estudia con detenimiento podrá reconocer en ella vestigios de una época en donde la ciudad de Mérida era centro de crecimiento, así como de experimentación de las vanguardias y del Movimiento Moderno.

La Iglesia Católica, el gobierno regional y la Universidad de Los Andes, todos ellos jugaron un rol importante en la modernización de la ciudad; siendo, además, promotores del proceso de adecuación del movimiento moderno en la ciudad de Mérida. Comprender esto es una necesidad y un primer paso para poder valorar y difundir las diferentes obras realizadas por seguidores de dicho movimiento en la ciudad, lo cual traerá como consecuencia la creación de propuestas que permitan preservar dichas edificaciones, así 
como reconocer edificaciones de valor que aún no gozan de declaratoria patrimonial, especialmente las ubicadas en la periferia del casco central. La realización de la Ruta Artística - Tipología Residencial, enfocada en las viviendas de corte moderno, permitirá a los usuarios hacer un recorrido diferente por el casco central de la ciudad, conocer al hombre merideño de mediados del siglo XX y cómo la ciudad de Mérida deja de ser la ciudad tradicional del damero americano, para convertirse en una ciudad que trata de asemejarse a la gran capital.

El trazado de la ruta turística tiene como objetivo, promover la puesta en valor del patrimonio moderno contemporáneo en la ciudad de Mérida a través de las edificaciones residenciales construidas durante dicho movimiento. Adicionalmente, se han planteado los siguientes objetivos específicos que permiten orientar las acciones:

1. Inventariar las edificaciones residenciales de corte moderno con declaratoria.

2. Ubicar las edificaciones residenciales de corte moderno con declaratoria sobre un plano de la ciudad con su dirección exacta.

3. Diseñar material editorial y web con información sobre edificaciones residenciales de corte moderno apto para el público en general.

4. Crear una campaña informativa y de participación comunitaria.

5. Generar un recorrido guiado que permita al usuario de la ruta compenetrarse con el patrimonio cultural.

6. Invitar a propietarios a realizar la ruta.

7. Promover la creación de convenios entre los entes competentes y los propietarios que estimulen a la buena conservación de los recursos patrimoniales de esta ruta.

8. Promover la creación de un programa de custodios del patrimonio para que a través de ellos se promocione la ruta y se ejecute la misma.

9. Promover la reactivación de la Policía Turística dentro de la ciudad para que, entre sus funciones, su personal sirva de facilitador de la ruta.

Inventario de los Recursos e Información:

1. Información contextual - La modernidad en Mérida y el crecimiento de la ciudad:

Es difícil imaginarse la ciudad de Mérida como una ciudad moderna o innovadora, ya que Mérida es una ciudad cuyas características geológicas y socio-culturales tienden a aislarla del mundo exterior y a ser más conservadora. Sin embargo, por ser sede de unas de las universidades más grandes y antiguas del país, la ciudad de Mérida siempre ha estado acompañada de visionarios que han buscado hacer de ella una ciudad al día.

Los principios de la Modernidad llegan a la ciudad de Mérida como consecuencia de un proceso de irradiación similar a la forma en que la modernidad llega a Venezuela, donde, en este caso, Caracas es el centro.

Es claro entender que los procesos de modernización de la ciudad de Mérida solían darse con cierto grado de retraso; sin embargo, entre 1895 y 1935 comienzan a verse los primeros signos de modernidad en la ciudad reflejados no solo en el comportamiento social, sino también en las diferentes transformaciones urbanas y arquitectónicas a lo largo de la ciudad.

Los primeros signos de modernidad en la ciudad de Mérida fueron muy tímidos; algunos de estos incluyeron la modificación de las plazas a un estilo más afrancesado, la incorporación de acueductos, fuentes de aguas, entre otros. Uno de los hechos que más marcan la llegada de la modernidad a la ciudad es la llegada del automóvil en 1916 (Febres-Cordero, 2003), trayendo como consecuencia el asfaltado de las calles de la ciudad.

La Universidad de Los Andes fue y ha sido el principal motor de modernización de la ciudad de Mérida, por lo que para finales del siglo XIX y principios del XX la ciudad tenía una atmosfera cultural muy particular abierta al arte y las ciencias. De igual forma, la ciudad también ha estado marcada siempre por una fuerte actividad religiosa. Cabe mencionar que, de hecho, la ULA tuvo como origen el Seminario (la ciudad fue elevada a la categoría de Sede Episcopal en el año 1785. Esto dio lugar a la creación de un seminario, que en 1811 se convertiría en la Universidad de Los Andes).

Entrados los años treinta, la ciudad sufre una aceleración en su proceso de modernización impulsado primordialmente por planes del gobierno nacional tales como la remodelación de la Plaza Bolívar. La inversión privada también comienza a refaccionar las fachadas de algunas de las casas coloniales que aún existían en la ciudad; "ello ocasiona algunos cambios en las fachadas urbanas de Mérida, pues en verdad resultan a la vista más elegantes y representativas" (op. cit., p. 88).

El rol de la Iglesia Católica en este período juega un papel importante, pues la cuadra arzobispal es refaccionada con un nuevo $\mathrm{Pa}$ lacio Arzobispal encargado por Monseñor Chacón al arquitecto Luis Bosetti. Pocos años más adelante, en preparación a la celebración del cuatricentenario de la ciudad, la construcción de una nueva Catedral es encargada al arquitecto Manuel Mujica Millán. Asimismo, se erigieron nuevos templos y otras edificaciones a lo largo de la cuadrícula urbana y de la meseta, como el caso del Colegio San Luis, que fue construido en la otra banda de la ciudad.

Febres- Cordero (2003) asegura que, en el caso particular de la ciudad de Mérida, entre los años 1895 y 1935, se estaría hablando de una etapa de anticipación, en los años cuarenta y cincuenta de una etapa de conformación de la modernidad arquitectónica, y que en la década de los sesenta y setenta "hay un proceso de consolidación de esa imagen de modernidad" (op. cit., p. 84) basado más en el estilo moderno.

Esta afirmación es posible comprobarla con tan solo darse un paseo por las diferentes obras arquitectónicas representativas de cada una de las épocas. El Edificio Rectoral y la antigua Facultad de Derecho de la ULA, el Hospital de Los Andes, la sede de la Policía y el pórtico de entrada al antiguo estadio, las escuelas estatales construidas por los planes especiales del gobierno central, entre otros, son ejemplos claros de esos primeros pasos hacia la modernidad arquitectónica en la ciudad.

La ciudad comienza a crecer hacia las periferias del casco central, marcando nuevos espacios urbanos, sobre la expansión de los ejes de circulación viales: avenida Don Tulio Febres - Cordero con la Facultad de Ingeniería de la Universidad de Los Andes; avenida Urdaneta con la construcción del Aeropuerto, Colegio de Médicos y otros; así como la avenida Gonzalo Picón, avenida 16 de Septiembre, y por último la avenida Universidad que ampliaba las avenida 1 y 2 . Es posible identificar en las propuestas arquitectónicas "la descomposición volumétrica y el programa funcional” (op. cit., p. 94).

Finalmente, una última característica de la puesta en práctica de los ideales del movimiento moderno en Mérida es la concepción de nuevos sectores habitacionales caracterizados por la presencia de casas quintas. Las primeras urbanizaciones que surgen en la ciudad de Mérida son El Encanto y Los Eucaliptos. 
2. Inventario de los Recursos y de la información:

De los 86 bienes inmuebles con declaratoria patrimonial, 38 son de tipología residencial; de estos 38 un total de 11 están referidos a la época moderna contemporánea - I y || etapa, en vista que para la III etapa de la época moderna contemporánea solo hay catalogada una edificación de tipo residencial unifamiliar; para que la misma forme parte de una ruta más especializada, se decidió incorporarla en el recorrido de esta ruta (Arnal, 2016).

La ruta ha sido referida a las construcciones de tipo residencial unifamiliar de corte moderno contemporáneo (I y II etapa) por lo que los recursos a ser utilizados son (Tabla 3):

\section{Creación de la Ruta:}

1. Accesibilidad y concentración de los recursos:

Los recursos patrimoniales utilizados en esta ruta se encuentran todos ubicados en el centro de la ciudad de Mérida. Están agrupados en tres sectores: Entre las calles 18 Fernández Peña y 20 Federación sobre la avenida 3, en la calle 30 Junín entre las avenida 4 Bolívar y avenida Don Tulio Febres Cordero, y en el sector Gloria Patrias, en la avenida 3 Independencia entre calles 34 Flores y calle 33 Boyacá.

Como esta ruta es parte de una propuesta de rutas culturales para Mérida, se propone como el inicio del recorrido (y de todos los recorridos) la Plaza Cruz Verde de Milla (Instituto de Patrimonio Cultural - IPC) / Plaza Chaplin (GM 110 - Gaceta Municipal 110). El recorrido es favorecido por la pendiente de la topografía de la meseta en el sentido hacia la Parque
Gloria Patrias (IPC) / Plaza Gloria Patrias (GM 110), siendo un recorrido de aproximadamente 26 cuadras.

Todos los recursos se encuentran ubicados sobre una vía pública; por lo tanto, el contacto con el exterior de la edificación es posible en cualquier momento. Ahora bien, la accesibilidad al interior de las edificaciones será limitada, ya que gran parte de ellas aún funcionan como residencias; y las demás son sedes de diferentes oficinas públicas y privadas. La ruta será realizada peatonalmente, aprovechando las cortas distancias y el sentido de la pendiente de la meseta sobre la cual está construida la ciudad.

2. Acceso a la documentación e información: (ver Tabla 3. Inventario de los recursos de la ruta propuesta).

\section{Creación de la Ruta:}

a. Recorrido, etapas y explicación: (ver Figura 1. Croquis de recorrido de la ruta y Tabla 4).

b. Fechas aptas para la realización de la Ruta: la ruta puede ser realizada los 365 días del año. Se recomienda el día domingo, ya que la dinámica de la ciudad disminuye, lo que permitirá al usuario disfrutar y contemplar cada edificación con mayor tranquilidad.

c. Temporalización: 100 minutos aproximadamente.

d. Imagen y Logotipo: siguiendo lo establecido por López (2016), se contactó a la empresa PG Idea, C.A., quien lleva casi 10 años trabajando con la creación de diseños alusivos a la ciudad de Mérida y Venezuela. Ello dio como resultado la siguiente propuesta (Figuras 2 y 3 ):

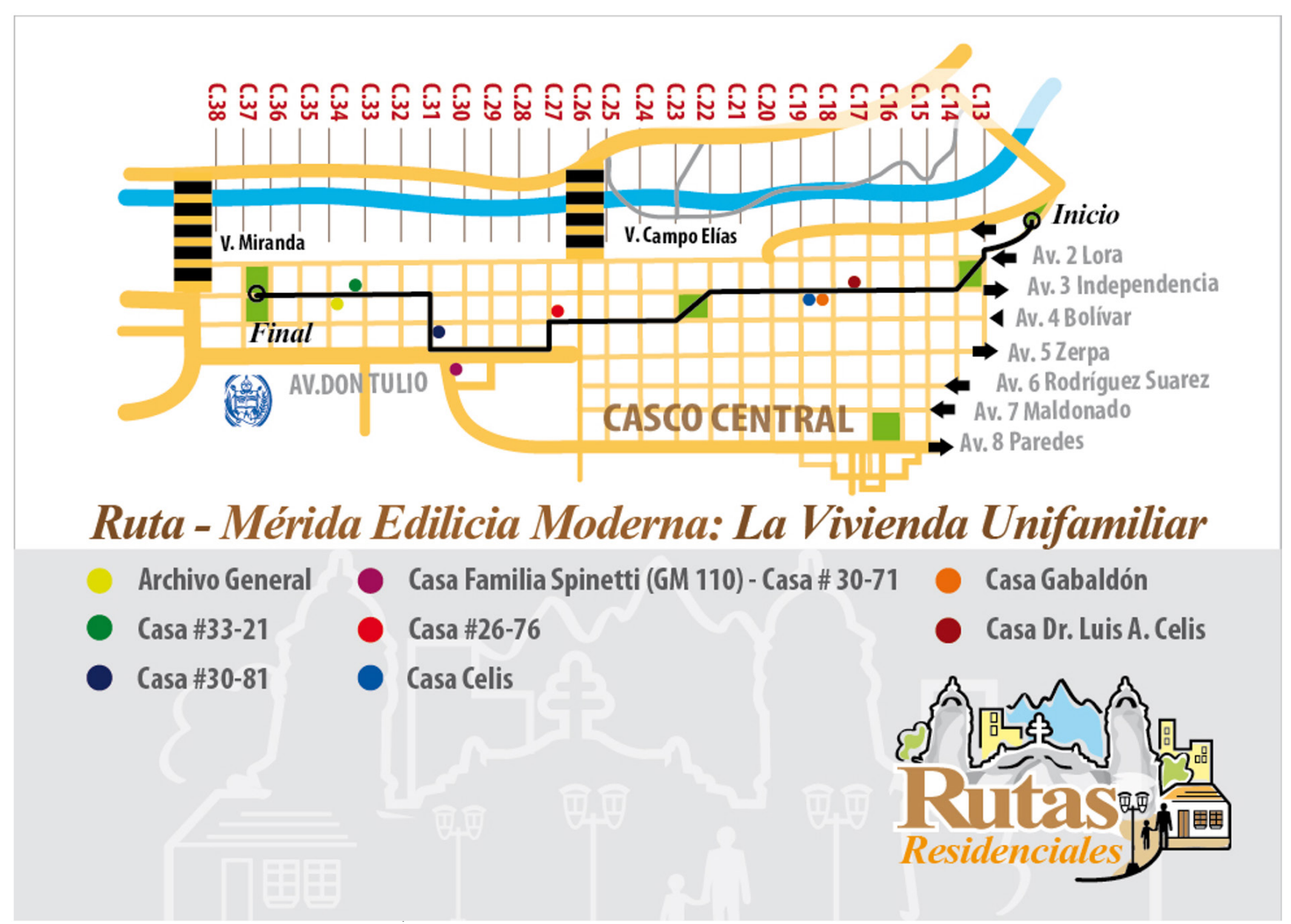

Figura 1. Croquis recorrido. 2016. 
Tabla 3. Inventario de recursos de la ruta propuesta. 2016.

\begin{tabular}{|c|c|c|c|c|}
\hline Recurso & Dirección & Descripción & Valor & Estilo \\
\hline $\begin{array}{l}\text { Archivo } \\
\text { General (IPC) / } \\
\text { Casa \#33-61 } \\
\text { (GM 110) }\end{array}$ & $\begin{array}{l}\text { Av. } 3 \text { Independencia } \\
\text { esquina con calle } \\
34 \text { Flores \#33-61 }\end{array}$ & $\begin{array}{l}\text { La casa en la que funciona el Archivo General de Mérida es la "Casa Elvira" o "Casa Va- } \\
\text { lecillo"; fue construida en } 1951 \text { siguiendo los parámetros de vivienda unifamiliar aislada } \\
\text { con retiros por los cuatro costados, marcando un cambio en el perfil urbano. Su sistema } \\
\text { constructivo es de concreto armado y paredes de bloques de arcilla. Diseñada original- } \\
\text { mente como una vivienda unifamiliar, sobresale en su fachada principal un balcón en } \\
\text { voladizo en la planta alta que hace de entrada con porche en la planta baja que conduce } \\
\text { a dos salones de recepción (Calderón - Trejo y Robinson, 2012). Originalmente era una } \\
\text { casa con ocho habitaciones, cocina, comedor y áreas de servicio. Actualmente todos } \\
\text { sus espacios han sido condicionados para el funcionamiento de las oficinas y salas del } \\
\text { archivo. }\end{array}$ & $\begin{array}{l}\text { Valor } \\
\text { Arquitectónico } \\
\text { Valor Singular - } \\
\text { Técnica }\end{array}$ & $\begin{array}{l}\stackrel{ }{\frac{1}{0}} \\
\frac{0}{2} \\
\sum\end{array}$ \\
\hline
\end{tabular}
archivo.

Es una edificación que se encuentra diagonal al Liceo Libertador. El trabajo realizado por Valor el arquitecto en la esquina le permite asumir una relación con su entorno urbano. Según Arquitectónico el IPC (2004), esta vivienda rompe con la continuidad en la fachada urbana de lo colonial. Av. 4 Bolívar esqui- Es una edificación que posee valor arquitectónico por la forma en la que incorpora el Casa \#26-76 na con calle 27 Ca- elemento semicircular en la esquina, el cual en planta baja hace de porche determinando rabobo \#26-76 el acceso de la vivienda mientras que en la planta superior es un balcón que continua a lo largo de toda la fachada de la av. 4 Bolívar. Según Febres-Cordero, citada por Iglesias (2005) el elemento semicircular introduce "referencias funcionalistas, produciendo un híbrido entre las referencias neocoloniales y funcionalistas" (p. 249).

\section{EI IPC (2004) declara que esta edificación posee un valor arquitectónico por la incorpo- Valor}

Av. 4 Bolívar es- ración del elemento semicircular en su fachada. Funcionalmente este elemento sirve de

Casa \#30-81 quina con Calle 31 acceso en planta baja y de balcón en la planta alta. Además se caracteriza por ser una Junín vivienda que incorpora los retiros, vivienda aislada, rompiendo así la continuidad de la fachada urbana en el damero (Febres-Cordero, 2003).

Arquitectónico

Av. 3 Independencia entre calles 33

$\begin{aligned} \text { Casa \#33-21 } & \begin{array}{l}\text { cia entre calles } 33 \\ \text { Boyacá y } 34 \text { Flores }\end{array}\end{aligned}$ \#33-21 costados.

\begin{tabular}{|c|c|}
\hline Casa Celis & $\begin{array}{l}\text { Calle } 19 \text { Cerrada } \\
\text { entre Av. } 3 \text { Indepen- } \\
\text { dencia y Av. } 4 \text { Bolí- } \\
\text { var, \# 3-39 }\end{array}$ \\
\hline
\end{tabular}

Av. 3 Independencia Casa Dr. Luis entre calles 17 y A. Celis 18 Fernández Peña $\# 17-26$

Av. 3 Independencia
Casa Gabaldón
$\begin{aligned} & \text { entre calles } 18 \text { Fer- } \\ & \text { nández Peña y } 19 \\ & \text { Cerrada }\end{aligned}$

Casa Familia Spinetti (GM 110) / Casa \# con Calle 31 Junín 30-71 \#30-71
Según Febres-Cordero (2003) en lo que a la vivienda dentro del damero en los años Valor cincuenta se refiere, ésta se caracteriza por la aparición de nuevos prototipos como lo Arquitectónico es el caso de la vivienda aislada. Esta vivienda se caracteriza por estar ubicada dentro del trazado urbano tradicional donde su emplazamiento incorpora el retiro por sus cuatro Según Febres - Cordero (2003), Manuel Mujica Millán plantea en esta vivienda una rein- Valor terpretación de la arquitectura de la colonia, razón por lo cual el acceso es una variación Arquitectónico del zaguán que integra la circulación vehicular y peatonal así como la transición entre lo público y lo privado. Esta reinterpretación también es percibida en el tratamiento de la planta baja en la fachada principal con una especie de añoranza del almohadillado clásico, que además permite enfatizar la horizontalidad en el volumen. Si bien la fachada es sencilla, la planta sigue un esquema funcional complejo a lo largo del lote rectangular sobre el cual está construido.

Según Febres-Cordero en lo que a la vivienda dentro del damero en los años cincuenta Valor se refiere, ésta se caracteriza por la aparición de nuevos prototipos como lo es el caso de Arquitectónico la vivienda aislada. En el caso particular de esta edificación se hace presente el retiro de frente rompiendo con la fachada urbana del sector.

El diseño de la vivienda permite que ésta se inserte en la escala y fachada urbana creando una continuidad del urbanismo en el centro de la ciudad. Es un ejemplo claro de la respuesta a esta tipología funcional y formal que presenta Mujica Millán en sus obras de viviendas en el centro. Es posible apreciar la reinterpretación moderna del zaguán con la inclusión de la circulación tanto peatonal como del vehículo. La volumetría está compuesta por dos elementos, uno de los cuales es resaltante, la torre, sin embargo existe el equilibrio entre la verticalidad y la horizontalidad en toda la obra (Febres - Cordero, Valor Singular Personajes 2003). La fachada presenta dos ventanas, que al iqual que el "zaquán", están rematadas por molduras realizadas en granito martillado.

Según Febres-Cordero (2003) en lo que a la vivienda dentro del damero en los años Valor cincuenta se refiere, ésta se caracteriza por la aparición de nuevos prototipos como lo Arquitectónico es el caso de la vivienda aislada. La vivienda de la familia Spinetti se caracterizó por ser Valor Singular una de las primeras viviendas aisladas sobre el eje vial Don Tulio Febres Cordero, además Personajes de ser una edificación diseñada siguiendo los parámetros más fieles de la arquitectura Valor Singular moderna.

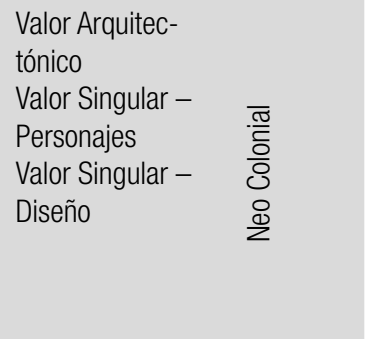

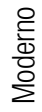
Diseño 
Tabla 4. Recorrido, estaciones y etapas de la ruta. 2016.

\begin{tabular}{|c|c|c|}
\hline $\begin{array}{c}\text { Recorrido / } \\
\text { etapa }\end{array}$ & Bien patrimonial & Explicación \\
\hline Inicio & $\begin{array}{l}\text { Plaza Cruz Verde de } \\
\text { Milla (IPC) / Plaza } \\
\text { Chaplin (GM 110) }\end{array}$ & $\begin{array}{l}\text { En esta primera estación el Guía realizará una pequeña introducción sobre la historia de la ciudad } \\
\text { de Mérida, y les dará la bienvenida a los usuarios a la ciudad histórica explicando de tal manera la } \\
\text { importancia de la Plaza en donde se encuentran y su significado. De esta forma, se invita al usuario a } \\
\text { hacerse consciente del viaje cultural que va a realizar. (Ver Arnal Sandia, 2016) } \\
\text { El Guía puede hacer mención de la Casa Villa del Carmen, que si bien no posee declaratoria patrimo- } \\
\text { nial, en ella se hace evidente la modernidad tras la incorporación de un retiro de frente y las líneas } \\
\text { puras de la fachada. }\end{array}$ \\
\hline $\begin{array}{l}\text { Recorrido - I } \\
\text { Tramo }\end{array}$ & & $\begin{array}{l}\text { El primer tramo del recorrido se realizará bajando por la Av. } 1 \text { Rodríguez Picón hasta llegar a la Calle } \\
13 \text { Colón. } \\
\text { A lo largo de este traslado, el Guía puede hacer referencia a cómo el centro de la ciudad de Mérida } \\
\text { tiene un tratamiento ecléctico y cómo la arquitectura se alterna entre lo tradicional y contemporánea, } \\
\text { ejemplificado en el contraste existente entre la Iglesia del Colegio San José de La Sierra y el Colegio } \\
\text { en sí. } \\
\text { En la calle } 13 \text { Colón cruza hacia la av. } 2 \text { Lora hasta encontrarse con el Parque Sucre o Plaza de Milla } \\
\text { (IPC) / Plaza Antonio José de Sucre (GM 110), la cual deberá ser atravesada diagonalmente hasta llegar } \\
\text { a la esquina sur de la plaza en la av. } 3 \text { Independencia; continuando el recorrido por dicha avenida. } \\
\text { Aquí el Guía, a medida que atraviesan la plaza, debe comentar características básicas de esta. } \\
\text { En este primer tramo del recorrido el Guía deberá haber aclarado el concepto de Arquitectura Moderna, } \\
\text { cómo nace el movimiento y su llegada a Mérida (Ver información contextual). }\end{array}$ \\
\hline
\end{tabular}

\begin{tabular}{|c|c|c|c|}
\hline I Estación & Casa Dr. Luis A. Celis. & \multirow[t]{2}{*}{ Ver Figura 3. Inventario de los recursos de la ruta propuesta } & vino tinto \\
\hline \|l Estación & Casa Gabaldón. & & naranja \\
\hline $\begin{array}{l}\text { Recorrido - II } \\
\text { Tramo }\end{array}$ & & Bajar por la av. 3 Independencia y cruzar en la calle 19 Cerrada. & \\
\hline III Estación & Casa Celis. & Ver Figura 3. Inventario de los recursos de la ruta propuesta & $\begin{array}{l}\text { azul } \\
\text { cobalto }\end{array}$ \\
\hline $\begin{array}{l}\text { Recorrido - III } \\
\text { Tramo }\end{array}$ & & $\begin{array}{l}\text { Retornar a la Av. } 3 \text { Independencia, bajar hasta la Plaza Bolívar; atravesarla diagonalmente para encon- } \\
\text { trarse con la esquina sur sobre la Av. } 4 \text { Bolívar, para continuar el recorrido hacia la calle } 27 \text { Carabobo. } \\
\text { El Guía debe hacer referencia a las generalidades de las líneas formales y estilísticas que caracteriza- } \\
\text { ron el Movimiento Moderno; puede apoyarse en las edificaciones vecinas de la Plaza Bolívar. }\end{array}$ & \\
\hline IV Estación & Casa \#26-76 & Ver Figura 3. Inventario de los recursos de la ruta propuesta & rojo \\
\hline $\begin{array}{l}\text { Recorrido - IV } \\
\text { Tramo }\end{array}$ & & $\begin{array}{l}\text { En la calle } 27 \text { Carabobo cruzar hacia la av. Don Tulio Febres Cordero. Caminar sobre la av. Don Tulio } \\
\text { Febres Cordero en sentido sur hasta llegar a la calle } 31 \text { Junín. } \\
\text { A lo largo de este recorrido el Guía mencionará el proceso de expansión de la ciudad de Mérida durante } \\
\text { los años 50, } 60 \text { y } 70 ; \text { el rol que tuvo la Universidad de Los Andes en este proceso y cómo el eje vial Don } \\
\text { Tulio Febres Cordero fue parte del crecimiento de la ciudad moderna (Ver Información contextual - La } \\
\text { modernidad en Mérida y el crecimiento de la ciudad). }\end{array}$ & \\
\hline V Estación & $\begin{array}{l}\text { Casa Familia } \\
\text { Spinetti (GM 110) / } \\
\text { Casa \#30-71 }\end{array}$ & Ver Figura 3. Inventario de los recursos de la ruta propuesta & fucsia \\
\hline $\begin{array}{l}\text { Recorrido V Tra- } \\
\text { mo }\end{array}$ & & Cruzar sobre la calle 31 Junín hacia la Av. 4 Bolívar. & \\
\hline VI Estación & Casa \#30-81 & Ver Figura 3. Inventario de los recursos de la ruta propuesta & índigo \\
\hline $\begin{array}{l}\text { Recorrido } \quad \mathrm{Vl} \\
\text { Tramo }\end{array}$ & & $\begin{array}{l}\text { Continuar sobre la calle } 31 \text { Junín hasta la av. } 3 \text { Independencia para cruzar sobre ella al sur hasta la } \\
\text { calle } 33 \text { Boyacá. En este tramo el guía puede hacer referencia al Ambulatorio El Llano. }\end{array}$ & \\
\hline VII Estación & Casa \#33-21 & & verde \\
\hline VIII Estación & $\begin{array}{l}\text { Archivo General (IPC) / } \\
\text { Casa \#33-61 (GM 110) }\end{array}$ & Ver Figura 3. Inventario de los recursos de la ruta propuesta & amarillo \\
\hline $\begin{array}{l}\text { Recorrido VII } \\
\text { Tramo }\end{array}$ & & Continuar bajando hasta el Parque Gloria Patrias (IPC) / Plaza Gloria Patrias (GM 110) & \\
\hline Fin & $\begin{array}{l}\text { La ruta culmina sobre } \\
\text { el Parque Gloria Patrias } \\
\text { (IPC) / Plaza Gloria Pa- } \\
\text { trias (GM 110) }\end{array}$ & $\begin{array}{l}\text { El Guía concluirá mencionando a la Mérida posterior a la I y II etapa de la época moderna (ver Arnal } \\
\text { Sandia, 2016) y cómo la ciudad comienza a crecer con la construcción de las diferentes urbanizaciones } \\
\text { tanto sobre el eje de la av. Urdaneta como sobre la av. Universidad (ver Información contextual - La } \\
\text { modernidad en Mérida y el crecimiento de la ciudad). }\end{array}$ & \\
\hline
\end{tabular}




\section{CONCLUSIONES}

La comunidad internacional, constantemente, invita a los ciudadanos a hacerse conscientes y responsables de su patrimonio, lo que conlleva a asumir una actitud y un accionar que permita conservarlo, difundirlo y preservarlo. Por tal razón, los trabajos recientes de los diferentes organismos se han centrado en la creación de propuestas que permitan preservar el patrimonio y simultáneamente la comunidad propietaria obtener beneficios socioeconómicos y culturales (ICOMOS, 2000).

Calvino (1983) afirma que la historia de la ciudad está contenida en la configuración de esta, y Peris (citado en Castillo, 2002) sostiene que la ciudad es un libro viviente capaz de narrar la historia, los modos de hacer y los estilos de sus antecesores. La continuidad entre el pasado del hombre y su presente está contenida en el libro viviente que es la ciudad, haciéndola parte del patrimonio y haciendo necesaria su valoración para preservarlo para las generaciones futuras (Arnal Sandia, 2016).

El gran auge que han tenido las propuestas de turismo cultural y el desarrollo de rutas culturales parte de lo expuesto anteriormente, ya que las mismas, puestas en práctica de manera correcta, pueden producir una sinergia casi perfecta de intercambio cultural y económico, donde el patrimonio es puesto en valor y preservado.

En el caso particular de Mérida, considerando la pluralidad cultural e histórica presente en ella, así como las actividades turísticas ya presentes en su dinámica cotidiana, proponer rutas culturales (como la presentada en este trabajo) es un primer paso para comenzar a incentivar la valoración y conservación del patrimonio entre los ciudadanos.

Esta investigación partió de un trabajo previo en el que se catalogaron las edificaciones con valor patrimonial de la ciudad de Mérida con base en el valor patrimonial y las características comunes de éstas, agrupando las mismas según hilos conductores establecidos por López (2006), creando el corpus para posibles rutas culturales, tal como la presentada en esta investigación, la ruta cultural: Mérida Edilicia Moderna: la vivienda unifamiliar.

Un total de ocho edificaciones ubicadas en la retícula con valor tradicional de la ciudad (casco central), forman parte de la ruta diseñada. Esta

\begin{tabular}{|c|c|c|}
\hline ELEMENTO & DESCRIPCIÓN & DISEÑO \\
\hline Logo & $\begin{array}{l}\text { Engloba los elementos esenciales y repre- } \\
\text { sentativos de Mérida como lo son su paisaje } \\
\text { (montañas), su sky - line marcada por la Ba- } \\
\text { sílica Menor, los edificios contemporáneos y } \\
\text { el merideño. }\end{array}$ & \\
\hline
\end{tabular}

Figura 2. Logo Rutas Culturales. 2016

Posee información general de la ciudad y un croquis con los 86 patrimonios inmuebles.
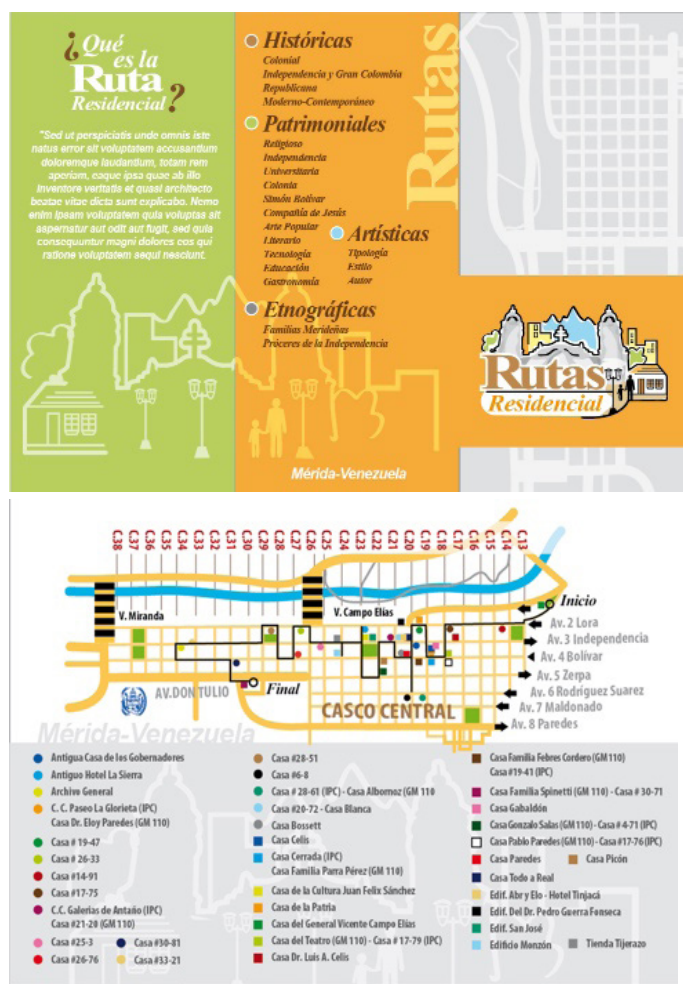

Figura 3. Tríptico Rutas Culturales. 2016 
ruta dará a conocer a quienes la recorren cómo el Movimiento Moderno impulsó la transformación de la ciudad tanto en su morfología como en el accionar de sus habitantes. Es, pues, una ruta con un recorrido conceptual diferente y potente, que permitirá a los usuarios conocer al hombre merideño de mediados del siglo XX y comprender en qué momento la ciudad de Mérida deja de ser la ciudad tradicional del damero americano para convertirse en una ciudad que emula a la gran capital sin perder por ello su personalidad propia.

\section{REFERENCIAS}

Alcaldía del Municipio Libertador. (2004). Gaceta Municipal Extraordinaria №. 110. Mérida, Venezuela: Autor.

Arnal Sandia, G. (2016). Ciudad, Historia y Patrimonio: Una Dinámica de Rutas Culturales para La Ciudad de Mérida. Tesis de Maestría. Universidad de Los Andes, Mérida, Venezuela.

Calvino, I. (1983). Las Ciudades Invisibles. Ciudad Editorial. http://www. siruela.com/archivos/fragmentos/Ciudadesindd.pdf

Carrera, G. (2006). Itinerarios y rutas: herramientas para la documentación y puesta en valor del patrimonio cultural. PH Boletín del Instituto Andaluz del Patrimonio Histórico, 60, 52 - 59. http:// www.iaph.es/revistaph/index.php/revistaph/article/viewFile/2256/2256

Castillo, M. (Comp.). (2002). Ciudades Históricas: conservación y desarrollo. Madrid, España: Fundación Argentaria.

Chacón, J. (2015). La Capilla de la Resurrección en el Seminario San Buenaventura de Mérida (Venezuela). Una experiencia de integración de las artes en el patrimonio arquitectónico. Actas del Congreso Internacional de Arquitectura Religiosa Contemporánea 4. https://doi.org/10.17979/aarc.2015.4.0.5127

Cherini Ramírez, S. (2007). Inscripciones Conmemorativas del Patrimonio Histórico Cultural del Casco Central de la Ciudad de Mérida - Venezuela. Boletín del Archivo Arquidiocesano de Mérida, Núm. 28, julio-diciembre, 2007, pp. 71-95. https://www.redalyc.org/pdf/691/69111714005.pdf

Febres - Cordero, B. (2003). La Arquitectura Moderna en Mérida. 1950 - 1959. Mérida, Venezuela. Talleres Gráficos, ULA.

Hernández, J. (2011) Los caminos del patrimonio. Rutas turísticas e itinerarios culturales PASOS. Revista de Turismo y Patrimonio Cultural, vol. 9, núm. 2, abril, 2011, pp. 225-236 Universidad de La Laguna El Sauzal (Tenerife), España. https://www.redalyc. org/pdf/881/88117284001.pdf

ICOMOS. (2000). Carta de Cracovia. http://ipce.mcu.es/pdfs/2000_ Carta_Cracovia.pdf

ICOMOS. (2014). Declaración de Florencia. http://www.icomos.org/ charters/culturalroutes_sp.pdf

Instituto del Patrimonio Cultural. (2007). Catálogo del Patrimonio Cultural de Venezuela 2004-2007. ME-12. Caracas, Venezuela: Instituto del Patrimonio Cultural.

López, M. (2006). Diseño y Programación de Itinerarios Culturales. PH Boletín del Instituto Andaluz del Patrimonio Histórico, 60, 20 - 33. http://www.iaph.es/revistaph/index.php/revistaph/article/ viewFile/2239/2239

Moreno Rodriguez, R. \& Bhaszar, J. F. (2020). Antonio Guzmán Blanco, precursor de la arquitectura moderna en Venezuela. Confluencias entre arte y política. Cuadernos de literatura del Caribe e Hispanoamérica, 31. http://investigaciones.uniatlantico.edu.co/revistas/index.php/cuadernos_literatura/article/ view/2543
Rengifo, J. (2006). Rutas Culturales y turismo en el contexto español. PH Boletín del Instituto Andaluz del Patrimonio Histórico, 60, 114 - 125. http://www.iaph.es/revistaph/index.php/revistaph/ article/view/2263

Torres, E. (2006). Rutas Culturales. Recurso, destino y producto turístico. PH Boletín del Instituto Andaluz del Patrimonio Histórico, 60, 84 - 97. http://webcache.googleusercontent.com/search?q=cache:cJnhbpE1snsJ:www.iaph.es/revistaph/index.php/ revistaph/article/download/2259/2259 